\title{
ANCAEE: A Novel Clustering Algorithm for Energy Efficiency in Wireless Sensor Networks
}

\author{
Ademola P. Abidoye ${ }^{1,3^{*}}$, Nureni A. Azeez ${ }^{2,3}$, Ademola O. Adesina ${ }^{1,3}$, Kehinde K. Agbele ${ }^{1,3}$ \\ ${ }^{1}$ Wireless Sensor Networks \& Natural Language Research Group, Cape Town, South Africa \\ ${ }^{2}$ Grid Computing \& Distributed Systems Laboratory, Cape Town, South Africa \\ ${ }^{3}$ Department of Computer Science, University of the Western Cape, \\ Cape Town, South Africa \\ E-mail: ${ }^{*}$ ademola.abidoye@gmail.com
}

Received July 18, 2011; revised August 19, 2011; accepted August 31, 2011

\begin{abstract}
One of the major constraints of wireless sensor networks is limited energy available to sensor nodes because of the small size of the batteries they use as source of power. Clustering is one of the routing techniques that have been using to minimize sensor nodes' energy consumption during operation. In this paper, A Novel Clustering Algorithm for Energy Efficiency in Wireless Sensor Networks (ANCAEE) has been proposed. The algorithm achieves good performance in terms of minimizing energy consumption during data transmission and energy consumptions are distributed uniformly among all nodes. ANCAEE uses a new method of clusters formation and election of cluster heads. The algorithm ensures that a node transmits its data to the cluster head with a single hop transmission and cluster heads forward their data to the base station with multi-hop transmissions. Simulation results show that our approach consumes less energy and effectively extends network utilization.
\end{abstract}

Keywords: Sensor Nodes, Clusters, Cluster heads, Wireless Sensor Networks, Base Station, Clustering Algorithms, Energy Efficiency

\section{Introduction}

Recent advances in communication technology have led to the development of intelligent, lightweight, low cost sensor nodes that cooperatively collect data from the place of deployment [1]. These nodes have the capability to communicate among themselves and with the base station. A sensor node consists of sensing, processing, communication, transceiver and power units [2]. These are used to acquire interested data, process, and communicate to other sensors within the networks usually, through radio frequency channel [3]. Wireless sensor networks (WSNs) have been used in many applications include home security, battle-field surveillance, monitoring movement of wild animals in the forest, earth movement detection, healthcare applications [4]. They can also be used in sensing ambient conditions such as light, sound, and temperature. Depending on the area of applications, sensor networks can be randomly distributed, for instance in military applications, sensor nodes can be randomly dropped from war-plane into the battlefield to monitor enemies' movement or manually placed.

Medical sensors can be manually placed in the hospital to monitor both the medical staff and patients inside the hospitals [5]. One of the main benefits of WSNs is their ability to operate autonomously in harsh environments where it may be dangerous or infeasible for human being to reach [6]. The nodes in WSNs are powered by batteries, it is expected that these batteries lasted for years before they can be replaced. Due to the cost and small size of the sensor nodes, they have been equipped with small batteries with limited energy source [7]. This has been a major constraint of wireless sensor nodes which limits their lifetime and affects utilization of the wireless sensor networks. To extend batteries' lifetime and networks utilization, constant changing the batteries when they run out of energy may not be practical, since these nodes in most cases are many (tens to thousands of sensor nodes), recharging the weaken batteries at all time may not be feasible. Therefore, there is a need to minimize energy consumption in WSNs. In recent time, many clustering algorithms have been proposed with different protocols 
by different researchers to prolong networks utilization. There still need to look for other techniques in which energy can be minimized in WSNs.

Clustering technique is one of the effective approaches used to save energy in WSNs [8]. Clustering means organizing sensor nodes into different groups called clusters. In each cluster, sensor nodes are given different roles to play, such as cluster head, ordinary member node, or gate way node. A cluster head $(\mathrm{CH})$ is a group leader in each cluster that collects sensed data from member nodes, aggregate, and transmits the aggregated data to the next $\mathrm{CH}$ or to the base station $[9,10]$. The role of ordinary member node is to sense data from the environment they deployed.

Gate-way nodes are nodes belonging to more than one clusters and their role is to transmit data between two clusters.

Furthermore, many different traditional clustering algorithms for wireless ad-hoc networks have been proposed by [11-13]. These clustering algorithms are not suitable for sensor networks because in ad-hoc networks, the primary concern is quality of service (QoS) and energy efficiency is the secondary. But in WSNs, the primary concern is the energy efficiency in order to extend the utility of the network [14].

However, Low Energy Adaptive Clustering Hierarchy (LEACH) Protocol was proposed by [15]. This protocol is one of the most famous hierarchical routing algorithms for energy efficiency in WSNs. Other algorithms developed thereafter were based on this algorithm.

The operation of LEACH protocol is divided into rounds. Each round consists of set-up phase and steadystate phase. During the set-up phase, sensor nodes are organized into different clusters based on the received signal strength and cluster heads are selected for each cluster as routers to the base station.

This algorithm saves energy, since only $\mathrm{CHs}$ are allowed to transmit data to the base station rather than all nodes. It allows $\mathrm{CHs}$ to rotate randomly to balance energy consumption of nodes in the networks. Basically, each node elects itself to be a $\mathrm{CH}$ in a given round. This decision is made by selecting a random number between 0 and 1. A node $\mathrm{V}$ becomes $\mathrm{CH}$ for the current round, if the number selected is less than set threshold value. The threshold formula is contained in [15]. LEACH achieves reduction in energy consumption 7 times compared with direct communication and between 4 to 8 times compared with minimum transmission energy (MTE) routing protocol [16]. Despite these benefits, LEACH suffers several shortcomings. CHs are not uniformly distributed in LEACH, CHs may be chosen from one part of the network. If this occurs, energy dissipation will be more than conventional protocols [17].
Moreover, considering a single round of $\mathrm{LEACH}, \mathrm{CH}$ selection based on probability will not automatically lead to minimum energy consumption during the steady state phase.

[26] proposed two-level LEACH (TL-LEACH) algorithm protocol. It was enhanced to the LEACH algorithm. They adopted the same method of cluster heads selection and clusters formation. The algorithm elected two sensor nodes in each cluster as cluster heads, one node as primary cluster head and the other as the secondary cluster head. Both the primary and secondary cluster heads can communicate with each other and secondary cluster heads communicate with nodes in their sub-clusters. Secondary cluster heads collect sensed data from the other nodes, perform data fusion and transmit it to the primary cluster heads. Primary cluster heads further perform data fusion on the received data and transmit it to the base station. This technique greatly reduces the number of data sent to the base station. However, the algorithm may not be energy efficient if the cluster heads are at distance from the base station. Finally, there is high probability of increase in overhead during the selection of primary and secondary cluster heads which will result to increase in energy consumption.

SEP is another clustering protocol, proposed by [18]. The main goal of the protocol is to use non-homogenous sensor nodes to distribute energy uniformly in WSNs. The protocol operation is similar to LEACH protocol, apart from method of cluster head selection is based on two different levels of energy. A node with the highest weight according to their different energy is elected as $\mathrm{CH}$. Subsequent $\mathrm{CHs}$ are elected using this process. This approach ensures that $\mathrm{CHs}$ are randomly selected and energy consumption is uniformly distributed among sensor nodes. The main objective of our research work is to develop an algorithm that will minimize communication distance among sensor nodes and prolong network utility.

\section{Clustering Algorithm}

Clustering is a good method in wireless sensor networks (WSNs) for effective data communication and towards energy efficiency [19]. It involves grouping of sensor nodes together, so that nodes communicate their sensed data to the CHs. CHs collect, aggregate and transmit the aggregated data to the processing centre called base station for further analysis [20]. Clustering provides resource utilization and minimizes energy consumption in WSNs by reducing the number of sensor nodes that take part in long distance transmission [21,22]. Cluster based operation consists of rounds. These involve cluster heads selection, cluster formation, and transmission of data to the base station. The operations are explained below. 


\subsection{Algorithm for Cluster Heads Selection}

In order for a node to become cluster head in a cluster the following assumptions were made.

- All the nodes have the same initial energy.

- There are S nodes in the sensor field.

- The number of clusters is $K$.

Based on the above assumptions, the average number of sensor nodes in each cluster is $M$ where

$$
M=\frac{s}{k}
$$

After $M$ rounds, each of the nodes must have been a cluster head $(\mathrm{CH})$ once.

We assigned each node a unique identifier $i, M_{i}$ for all $i=0,1,2,3,4, \cdots S-1$

Variable $i$ is used to test whether it is the turn of a node to become a $\mathrm{CH}$.

Originally, all nodes are the same, i.e. there is no CHs in each cluster, $j=0$ where $j$ is CHs counter.

A node $q$ is selected among all nodes and continuously executes the following steps:

Firstly, $q$ increments $i$ by 1 and check if $i$ is even, if yes that node is selected as the $\mathrm{CH}$ for that round and announces its new position to all member nodes in the cluster.

Else if $i$ is odd, it cannot be a $\mathrm{CH}$ for that round, it will wait for the next round and be ready to receive advertisement message from the new $\mathrm{CH}$.

A predetermined value is set (threshold value) for the new $\mathrm{CH}$ to transmit for that round.

When the value has reached, $j$ will be incremented by 1 and the process of selection of new $\mathrm{CH}$ begins. It tests if the following two conditions hold.

- That a sensor node has not become cluster head for the past $(1 / p)-1$ rounds [25].

- That the residual energy of a node is more than the average energy of all the sensor nodes in the clustering.

Thus, the probability of a node becoming new cluster head is given as

$$
P_{i}=\frac{E_{\text {rem }}(i) * K}{E_{\text {avg }}(i) * M}
$$

where $E_{\text {rem }}$ is the remaining energy in node (i), $E_{\text {avg }}$ is the average energy of all the nodes in a cluster.

It continues until $j=K$. The algorithm stops when $j=K$. The new CHs collect sensed data from member nodes, aggregate them, and transmit the compressed data to the next cluster head or base station.

\subsection{Cluster Formation}

The next step in the clustering phase is cluster formation after CHs have been elected. Below gives the description of new cluster formation.

Step 1: The new cluster heads elected above broadcast advertisements (ADV) message to all non-cluster nodes in the network using Carrier Sense Multiple Access (CSMA) MAC Protocol.

Step 2: Each sensor node determines which clusters it will join, by choosing $\mathrm{CH}$ that requires minimum communication energy.

Step 3: Each non-cluster node uses CSMA to send message back to the CHs informing them about the cluster it wants to belong.

Step 4: After $\mathrm{CHs}$ have received messages from all nodes, Time Division Multiple Access (TDMA) scheduling table will be created and send it to all nodes. This message contains time allocated to each node to transmit to the $\mathrm{CH}$ within each cluster.

Step 5: Each sensor node uses TDMA allocated to it to transmit data to the $\mathrm{CH}$ with a single- hop transmission and switch off its transceiver whenever the distance between the node and $\mathrm{CH}$ is more than one hop to conserve energy.

To avoid a single node transmitting data multiple times in one round, we set a threshold value $G$. $G$ is the total time of all nodes in the cluster forwarding their data to the $\mathrm{CH}$ in one round.

Step 6: CHs will issue new TDMA slots to all nodes in their clusters when allocated time for $G$ has elapsed, for each node to know exact time it will transmit data to avoid data collision during transmission that can increase energy consumption.

Step 7: $\mathrm{CH}$ transceiver is always turn-on to receive data from each node in its cluster and prepare them for inter-clusters transmission.

Inter-cluster transmission is of two types: single hop and multi-hop [23,24]. We adopted multi-hop transmission in order to save more energy during inter-cluster transmission.

\subsection{Steady State Phase}

After all data has been received, the $\mathrm{CH}$ performs data fusion function by removing redundant data and compresses the data into a single packet. This packet is transmitted to the base station via multi hops transmission. After a certain period which is calculated in advance, the next round starts with the election of new CHs using our initial algorithm as described in Section 2.1 above and formation of new clusters as explained in Section 2.2.

\section{Simulation Results and Analysis}

The main aim of this experiment is to extend the network 
lifetime by minimizing communication distance during transmission. In order to evaluate the performance of our new clustering algorithm (ANCAEE), we simulated LEACH, TL-LEACH protocol and ANCAEE using MATLAB. To see the level of energy saving our protocol can achieve, we used 100 sensor nodes randomly distributed between $(0,0)$ and $(100,100) \mathrm{m}$ with base station set at a distance $(x=50, y=350) \mathrm{m}$ as shown in Figure 1.

Simple Radio energy dissipation model used for the experiment was adapted from [15].

From the radio model, the energy expended by the radio to transmit $k$ bits of data to distance $d$ is given by

$$
\begin{aligned}
E_{\text {tx }}(k, d) & =E_{\text {elec }} * k+E_{\text {amp }} * k \\
& =E_{\text {elec }} * k+\varepsilon_{f s} * d^{2} * k \text { where } d d_{0} \\
& =E_{\text {elec }} * k+\varepsilon_{m p} * d^{4} * k d \geq d_{0}
\end{aligned}
$$

To receive $k$ bits of data is given by

$$
E_{r x}(k)=E_{\text {elec }} * k
$$

$E_{\text {elec }}$ is the energy expended per bit to run the transmitter or receiver circuit as shown in Equations (3) and (4).

Energy dissipation by transmitter amplifier depends on distance $d$ between sender and receiver. For this experiment, both the Free State ( $d^{2}$ power loss) and the Multipath Fading ( $d^{4}$ power loss) Models were used.

We set threshold value $d_{0}$ for the distance between sender (sensor nodes) and the receiver (Cluster heads and Base station).

If the distance $d$ is less than $d_{0}$, Free State model is used to know energy dissipation by the transmitter electronics else, Multipath Fading model is used.

The parameters used for the test and the respective values are given in Table $\mathbf{1}$.

Sensor field containing 100 nodes is partitioned into five clusters; each cluster contains a cluster head and member nodes. Red nodes represent cluster heads and

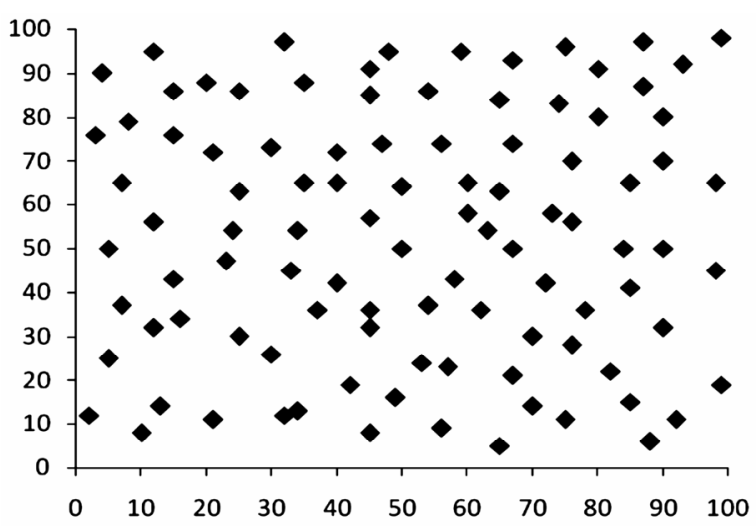

Figure 1. Sensor nodes randomly distributed.
Table 1. Simulation parameters.

\begin{tabular}{lc}
\hline \multicolumn{1}{c}{ Parameters } & Values \\
\hline Number of Sensor Nodes & 100 \\
Network Dimension & $100 \mathrm{~m} \times 100 \mathrm{~m}$ \\
Nodes initial energy $(K)$ & $0.5 \mathrm{~J}$ \\
Transmitter circuitry dissipation $\left(E_{\text {ele }}\right)$ & $50 \mathrm{~nJ} / \mathrm{bit}$ \\
Amplifier dissipation multipath $\left(\varepsilon_{m p}\right)$ & $0.0015 \mathrm{pJ} / \mathrm{bit} / \mathrm{m}^{4}$ \\
Data packet size & $100 \mathrm{bytes}$ \\
Amplifier dissipation free state $\left(\varepsilon_{f s}\right)$ & $10 \mathrm{pJ} / \mathrm{bit} / \mathrm{m}^{2}$ \\
Broadcast size & $25 \mathrm{bytes}$ \\
Distance between sensor field and Base-station & $x=50, y=350$ \\
\hline
\end{tabular}

black nodes represent member nodes. Each of the nodes is assigned with unique identifier (ID) as shown in Figure 2.

We compared the performances of our algorithms with LEACH and TL-LEACH algorithms. We are interested in the number of rounds when the first node dies and time when the last node dies.

From the simulation result shown in Figure 3, it was observed that the first node dies in LEACH and TLLEACH after about 135 and 148 rounds respectively and while in ANCAEE first node dies after about 185 rounds. Furthermore, the last dies in LEACH after about 640 rounds and 710 rounds in TL-LEACH while last node dies after 800 rounds in ANCAEE.

This simulation result shows that ANCAEE algorithm extends battery life time thus, prolongs sensor network utilization.

Figure 4 shows number of sensed data received at base station (BS) over transmission rounds. Our algorithm

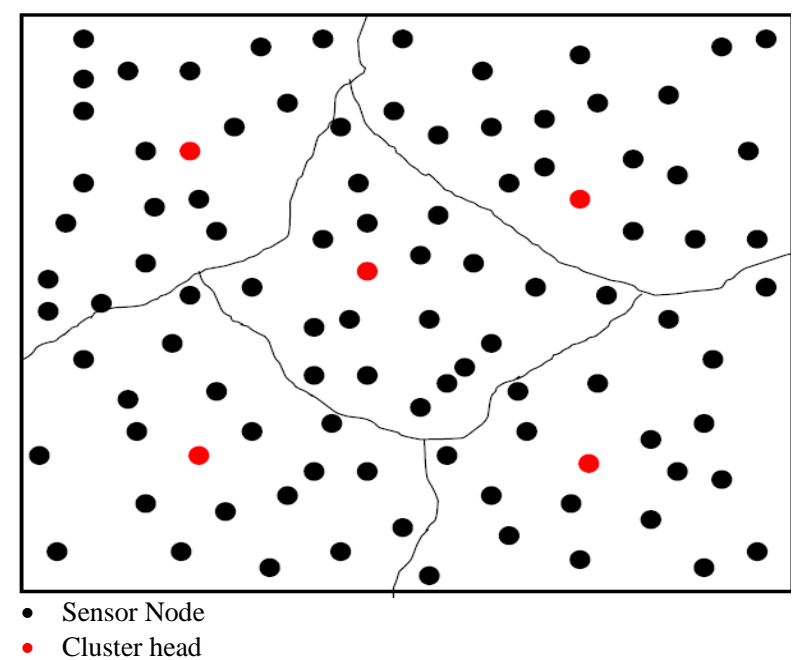

Figure 2. Cluster formation. 


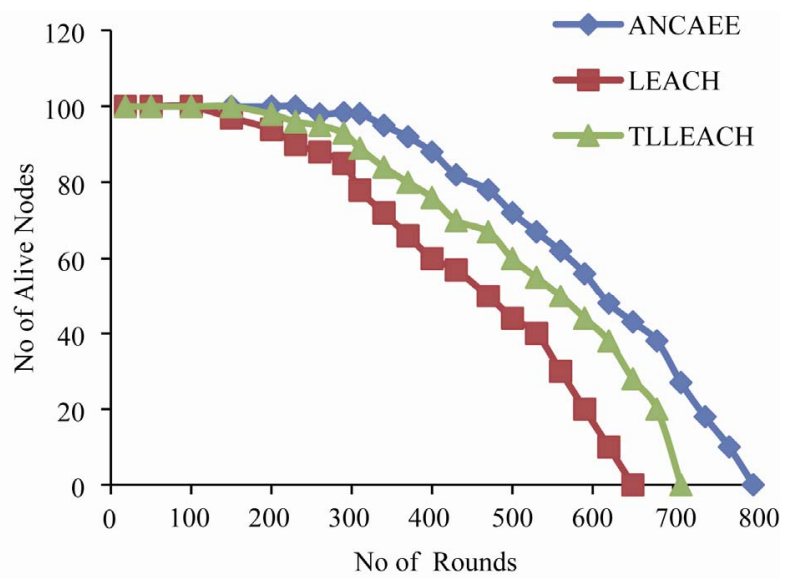

Figure 3. Number of nodes still alive over no of rounds.

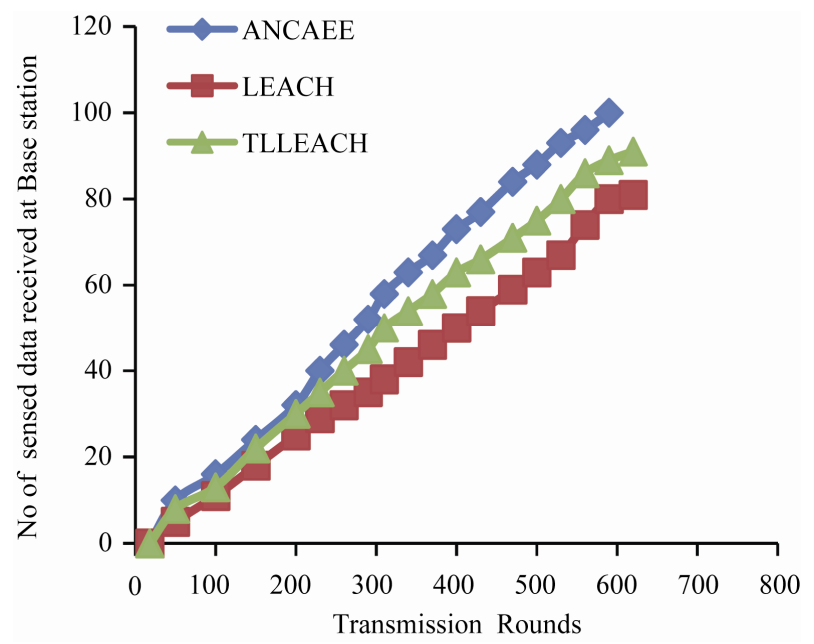

Figure 4. Number of data received at base station.

selects new cluster heads (CHs) based on remaining energy of each node. It ensures that nodes with high residual energy are selected as $\mathrm{CHs}$ within a set value. The algorithm transmits more data to the base station compare with LEACH and TL-LEACH algorithms with minimum energy dissipation.

\section{Conclusions}

In this paper, we introduced new method of cluster heads selection and clusters formation algorithm. Our algorithm, ANCAEE partitioned the sensor field into different clusters and elects a node as the cluster head for each cluster. Each node within the cluster sends its data to the cluster head with single hop transmission and cluster heads receive, aggregate the data and transmit to the base station via multi-hops transmission. This method conserves energy dissipation of sensor nodes in the clusters.

Simulation results show that using this technique, ANCAEE saves more energy than LEACH and TL-
LEACH protocols due to short range data transmission of sensor nodes and election of cluster heads based on residual energy of each node. The objective of this algorithm is achieved of consuming less energy, distributing energy consumption equally among all nodes and finally, extends the network lifetime.

\section{References}

[1] W. Wang, et al., "CEDCAP: Cluster-Based energy-Efficient Data Collecting and Aggregation Protocol for WSNs," Journal of Information Technology Research, Vol. 3, No. 2, 2011, pp. 93-103.

[2] D. Zhicheng, Z. Li, B. Wang and Q. Tang, “An Energy-Aware Cluster-Based Routing Protocol for Wireless Sensor and Actor Network," Journal of Information Technology, Vol. 8, No. 7, 2009, pp. 1044-1048. doi:10.3923/itj.2009.1044.1048

[3] Azat Rozyyev, H. Hasbullah and F. SubhanIndoor, "Indoor Child Tracking in Wireless Sensor Network Using Fuzzy Logic Technique,” Research Journal of Information Technology, Vol. 3, No. 2, 2011, pp. 81-92. doi:10.3923/rjit.2011.81.92

[4] I. F. Akyildiz, W. Su, Y. Sankarasubramaniam and E. Cayirci, “A Survey on Sensor Networks," IEEE Communication Magazine, Vol. 40, No. 8, 2002, pp. 102-114.

[5] J. N. Al-Karaki and A. E. Kamal, "Routing Techniques in Wireless Sensor Networks: A Survey," IEEE Wireless Communications, Vol. 11, No. 6, 2004, pp. 6-28. doi:10.1109/MWC.2004.1368893

[6] K. Sohrabi, J. Gao, V. Ailawadhi and G. J. Potie, "Protocols for Self-Organization of a Wireless Sensor Network," IEEE Personal Communications, Vol. 7, No. 5, 2000, pp. 16-27.

[7] A. Gao, W. Wei and X. Xiao, "Multiple Hash SubChains: Authentication for the Hierarchical Sensor Networks," Information Technology Journal, Vol. 9, No. 4, 2010, pp. 740-748.

[8] X. Guan, W. H. Yang and D. G. Bi, "EEHCA: An Energy-Efficient Hierarchical Clustering Algorithm for Wireless Sensor Networks," Information Technology Journal, Vol. 7, No. 2, 2008, pp. 245-252.

[9] J. Yang and D. Zhang, “An Energy-Balancing Unequal Clustering Protocol for Wireless Sensor Networks," Information Technology Journal, Vol. 8, No. 1, 2009, pp. 57-63. doi:10.3923/itj.2009.57.63

[10] D. Wei, S. Kaplan and H. A. Chan, "Energy Efficient Clustering Algorithms for Wireless Sensor Networks," Proceeding of the IEEE International Conference on Communications Workshops, 19-23 May 2008, Beijing, pp. 236-240.

[11] Z. Li, R. Li, Y. Wei and T. Pei, "Survey of Localization Techniques in Wireless Sensor Networks," Information Technology Journal, Vol. 9, No. 8, 2010, pp. 1754-1757.

[12] W. Wang, et al., "Cross Layer Design and Implementation for Balancing Energy Efficiency in Wireless Sensor Networks," Information Technology Journal, Vol. 6, No. 
2, 2007, pp. 648-655.

[13] Y. Xu, J. Heidemamij and D. Estrin, "Geography-Informed Energy Conservation for Ad Hoc Routing," Proceedings of the ACM/IEEE 7th Annual International Conference on Mobile Computing and Networking, Rome, 16-21 July 2001, pp. 70-84.

[14] J. Chen, J. Fan, X. Cao and Y. Sun, "GRFR: Greedy Rumor Forwarding Routing for Wireless Sensor/Actor Networks," Information Technology Journal, Vol. 7, No. 4, 2008, pp. 661-666.

[15] W. B. Heinzelman, A. P. Chandrakasan and H. Balakrishnan, "An application-Specific Protocol Architecture for Wireless Microsensor Networks,” IEEE Transactions on Wireless Communications, Vol. 1, No. 4, 2002, pp. 660-670. doi:10.1109/TWC.2002.804190

[16] K. Akkaya and M. Younis, "A Survey on Routing Protocols for Wireless Sensor Networks," Ad Hoc Networks, Vol. 3, No. 3, 2005, pp. 325-349. doi:10.1016/j.adhoc.2003.09.010

[17] M. Ahmad, M. Habib, M. Z. Shah, F. Ullah and S. Hussain, "Energy Aware Uniform Cluster-Head Distribution Technique for Hierarchal Wireless Sensor Networks," International Journal of Computer Science and Network Security, Vol. 10, No. 10, 2010, pp. 97-103.

[18] G. Smaragdakis, I. Matta and A. Bestavros, "SEP: A Stable Election Protocol for Clustered Heterogeneous Wireless Sensor Networks," 2nd International Workshop on Sensor and Actor Network Protocols and Applications, Boston, 22 August 2004, pp. 56-66.

[19] K. Zhou, L. Meng, Z. Xu, G. Li and J. Hua, “A Dynamic Clustering-Based Routing Algorithm for Wireless Senor Networks,” Information Technology Journal, Vol. 7, No. 4, 2008, pp. 694-697.
[20] A. D. Amis, R. Prakash, T. H. P. Vuong and D. T. Huynh, "Max-Min D-Cluster Formation in Wireless Ad-Hoc Networks," Proceedings of the IEEE 9th Annual Joint Conference of the IEEE Computer and Communications Societies, Tel Aviv, 26-30 March 2000, pp. 32-41.

[21] Y. He, W. S. Yoon and J. H. Kim, "Multi-level Clustering Architecture for Wireless Sensor Networks," Information Technology Journal, Vol. 5, No. 1, 2006, pp. 188-191. doi:10.3923/itj.2006.188.191

[22] W. Liu and J. Yu, "Energy Efficient Clustering and Routing Scheme for Wireless Sensor Networks,” Proceeding of the IEEE International Conference on Intelligent Computing and Intelligent Systems, Shanghai, 20-22 November 2009, pp. 612-616. doi:10.1109/ICICISYS.2009.5358113

[23] X. Hong, G. Mario and C. C. Chiang, "A Group Mobility Model for Ad Hoc Wireless Networks," Proceedings of the 2nd ACM International Workshop on Modeling Analysis and Simulation of Wireless and Mobile Systems Seattle, New York, 20 August 1999, pp. 53-60.

[24] G. Yan and J. Xu, “A Clustering Algorithm in Wireless Networks," Proceeding of the International Conference on Multi Media and Information Technology, Three Gorges, 30-31 December 2008, pp. 629-632.

[25] M. J. Handy, M. Haase and D. Timmermann, “Low Energy Adaptive Clustering Hierarchy with Deterministic Cluster-Head Selection," 4th IEEE International Workshop on Mobile and Wireless Communications Network, Stockholm, 9-11 September 2002, pp. 368-372.

[26] V. Loscri, G. Morabito and S. Marano, “A Two-Level Hierarchy for Low-Energy Adaptive Clustering Hierarchy," 62nd IEEE Vehicular Technology Conference, Vol. 3, No. 2, 2005, pp. 1809-1813. 